\title{
Arthur I. Miller: The artist in the machine: the world of Al-powered creativity
}

\author{
The MIT Press, 2019, 32 colour ills, 432 pp, ISBN: 9780262354592 ; \\ 9780262539623
}

\section{Anna Olszewska ${ }^{1}$ (D)}

Received: 6 May 2020 / Published online: 2 June 2020

(c) Springer Science+Business Media, LLC, part of Springer Nature 2020

Recent advancements in deep learning have reinvigorated interest at the intersection of art, creativity and machine learning. Current interest in creative machines captivates diverse audiences, thus rekindling the 1960's fascination with cybernetics. Readers are invited to choose between various types of studies introducing the issue of AI creativity, such as "Computational Creativity: The Philosophy and Engineering of Autonomously Creative Systems" edited by Tony Veale and Amílcar Cardoso [1]. There are also art history insights on the coexistence of AI and the arts, such as the recently published catalogue of the retrospective exhibition "Artistes \& Robots" held in Paris, curated by Jérôme Neutres and Laurence Bertrand-Dorléac [2]. In The artist in the machine, Arthur I. Miller responds to this new wave of fascination with computer creativity with a book addressed to a general readership.

Although it is a work of non-fiction, The Artist in the Machine unravels like a James Bond scenario. The plot dives straight into the world of art, ushers us through the headquarters of tech companies only to finally hurl us into a posthuman vision of machine-inhabited planets. In the story, creative geniuses are characterized as moderately nefarious, of eclectic background, who live complicated lives and entangle themselves into disastrous liaisons. Picasso figures here as a patron saint of the artistic domain, while Poincaré and Einstein typify ingeniousness in mathematics and physics. Throughout this account, Silicon Valley barons and a long-awaited breed of artist-engineers incarnate the myth of modern avantgarde.

The book aims at confirming that, as a consequence of AI developments, machine creativity does exhibit traits customarily assigned to humans. To justify this claim, it explores the domains of visual arts, music, literature and theatrical performance. Arguments are drawn from numerous histories of projects that employed AI for innovative purposes. The book introduces a distinction

Anna Olszewska

aolsz@agh.edu.pl

1 Faculty of Humanities, AGH University of Science and Technology, Gramatyka 8a,

30-046 Kraków, Poland 
between instances of AI creativity and the tradition of the twentieth century preprogrammed computer arts. The lead concept focuses on the creativity traits that reside in the maker, and it is assumed that they emerge from biographies of individuals recognized as geniuses. Despite a variety of insights into the matter, it is Miller's own set of hallmarks of creativity that becomes the reference point for the concluding evaluation of the machine's potential.

Similarly to his 2014 study [3], The artist in the machine is in its core part based on his interviews with representatives of the third culture. In the current selection the reader will be acquainted with members of Google, Ars Electronica and ZKM networks. Among them one finds engineers exploring AI theory as well as those who develop new deep learning architectures (in particular ones dealing with generative modelling). They are presented side by side with visual artists, composers, poets and playwrights who are either involved in digital media or who use AI as a tool for their creations.

The text has been structured into three components. The introduction outlines the concept of creativity that prepares the ground for the final evaluation of the machine's potential. Biographical accounts of the most distinguished scientists and artists of the twentieth century bind creativity with notions of genius, novelty, and independence.

The following parts (II-V) describe programmers, artists and scientists who have introduced AI generated artefacts into the art domain. Concise chapters enumerate biographical details of the interviewees, outline the history of research behind the most recognized achievements, and present the interviewees assertions in matters of creativity. The aims and goals of the discussed projects are miscellaneous and inclusive, with their fields of activities varying from machine powered design to speculative studies in humanities. Among numerous instances, one can find achievements in generative modelling (i.e. Ian Goodfellow's history of GANs, Alexander Mordvintsev's experiments with Deep Dream and Phillip Isola's ideas behind Pix2Pix), software development (i.e. Douglas Eck's introduction to Magenta), artistic expression (i.e. by Allison Parrish, Anna Ridler and the Obvious Collective) and digital archives mashups (i.e. Refik Anadol and Mario Klingerman).

The last part introduces endeavours in deep learning theory based on interviews with Jason Yosinski and Mark Riedl, as well as ideas on the evaluation of machine creativity suggested by Graeme Ritchie, Geraint Wiggins and Anna Jordanous. Possible drivers of creativity, outlined by Margaret Boden, are supplemented with ideas such as affect computing (Rosalind Picard) and machine awareness, attention and consciousness (Michael Graziano). The concluding chapter (Chapter 46) presents the proof of machine creativity by adapting human traits of creativity to AI.

The book's major strength lies with its characters. Much effort has been made to collect all the makers in one volume, present their statements, and affirm their position within the firmament of the third culture. On the other hand, what feels missing is an attempt toward combining the interviewees' insights into a collective concept for machine creativity. A reflexive approach is essential to outlining theoretical account on socially established norm. Such a concept of creativity would expose ideas at stake in the current race for artificial life. 
The artist in the machine provides an update on the latest art and technology developments. The core chapters supply points of reference for those who wish to explore the 2010s tech creativity. The book might also serve as an encouragement to reflect over human practices in AI. Reading through a such a plethora of statements provokes inquiry into conditions under which we agree to deep learning operating autonomously and thus allow machines to promote creativity for their own sake.

\section{References}

1. T. Veale, F.A. Cardoso (eds.), Computational Creativity: The Philosophy and Engineering of Autonomously Creative Systems (Springer, Dordrecht, 2019)

2. J. Neutres, L. Bertrand-Dorléac, Catalogue de l'exposition "Artistes et robots" au Grand Palais, Galeries Nationales 5 April to 9 July 2018. (RMN-Grand Palais, Paris, 2018)

3. A.I. Miller, Colliding Worlds: How Cutting-Edge Science Is Redefining Contemporary Art (W. W. Norton \& Company, New York, 2014)

Publisher's Note Springer Nature remains neutral with regard to jurisdictional claims in published maps and institutional affiliations. 